Communications in Physics, Vol. 14, No. 2 (2004), pp. 119-125

\title{
HILLERY-TYPE AMPLITUDE SQUEEZING IN LINEAR AND NONLINEAR FAN-STATES
}

\author{
TRUONG MINH DUC \\ Physics Department, Hue University
}

\begin{abstract}
Squeezing properties of the Hillery-type $N$-powered amplitude are investigated in the linear and nonlinear fan-state. For a given $k$, squeezing may appear to the even power $N=2 k$ and the number of directions along which the Nth-powered amplitude is squeezed is exactly equal to $N$, in both linear (the light field) and nonlinear (the vibrational motion of the trapped ion) fan-states.
\end{abstract}

\section{INTRODUCTION}

The squeezed state is a nonclassical state, which has been known for a long time (see, e.g., a recent review in [1]). This state is frequently used in quantum optics and in other branches of quantum physics. Squeezed states have attracted much interest thanks to their potential applications in communication networks, detecting extremely weak fields, waveguide tap [2] and quantum information theory [3-7]. The conventional squeezed states [8] have been generalized to different types of higher-order. The first type of higher-order amplitude squeezing was given by Hong and Mandel [9]. The second type of higher-order squeezing which is qualitatively different from that by Hong and Mandel, was defined by Hillery [10] and then developed further by many authors (see, e.g., [11-13]). The Hong-Mandel-type $N$-order amplitude squeezing has recently been studied in the fanstates $|\xi ; 2 k, f\rangle_{F}$ which is introduced in [14] as a linear superposition of $2 k 2 k$-quantum nonlinear coherent states in the phase-locked manner. In this paper, we study properties of the Hillery-type $N$-powered amplitude squeezing in the fan-states. We call these states linear if $f=1$ and nonlinear if $f \neq 1$. In the nonlinear case $f$ is an arbitrary nonlinear operator-valued function of $\hat{n}=a^{+} a$ with $a\left(a^{+}\right)$the boson field annihilation (creation) operator. Keeping the notation as in [15], the normalized fan-state is defined as

$$
|\xi ; 2 k, f\rangle_{F}=D_{k}^{-1 / 2} \sum_{q=0}^{2 k-1}\left|\xi_{q} ; 2 k, f\right\rangle
$$

where $k=1,2,3, \ldots ; \xi_{q}=\xi \exp \left(\frac{i \pi q}{2 k}\right)$ with $\xi$ a complex number,

$$
D_{k}=D_{k}\left(|\xi|^{2}\right)=\sum_{m=0}^{\infty} \frac{|\xi|^{4 k m}\left|J_{k}(m)\right|^{2}}{(2 k m) !\left[f(2 k m)(!)^{2 k}\right]^{2}},
$$

with $J_{k}(m)=\sum_{q=0}^{2 k-1} \exp (i \pi q m)$, and

$$
\left|\xi_{q} ; 2 k, f\right\rangle=\sum_{n=0}^{\infty} \frac{\xi_{q}^{2 k n}}{\sqrt{(2 k n) !} f(2 k n)(!)^{2 k}}|2 k n\rangle
$$


with $|2 k n\rangle$ a Fock state. The state $\left|\xi_{q} ; 2 k, f\right\rangle$ is a sub-state of the multi-quantum nonlinear coherent states [16-19], the eigenstates of the operator $a^{2 k} f(\hat{n})$ with $k$ a positive integer and $f$ an arbitrary real nonlinear operator-valued function of $\hat{n}$. The notation $(!)^{2 k}$ is understood as follows

$$
f(p)(!)^{2 k}=\left\{\begin{array}{ll}
f(p) f(p-2 k) f(p-4 k) \ldots f(q) & \text { if } p \geq 2 k ; 0 \leq q<2 k \\
1 & \text { if } 0 \leq p<2 k
\end{array} .\right.
$$

The N-powered amplitude squeezing is associated with the operator $Q_{N}(\varphi)$ of the form

$$
Q_{N}(\varphi)=\frac{1}{2}\left(a^{N} e^{-i N \varphi}+a^{+N} e^{i N \varphi}\right)
$$

with $\varphi$ an angle determining the direction of $\left\langle Q_{N}(\varphi)\right\rangle$ in the complex plane and the operators $a, a^{+}$obeying the commutation relation $\left[a, a^{+}\right]=1$. According to [12-13], a state $|\ldots\rangle$ is said to be Hillery-type amplitude $N$ th power squeezed in the direction $\varphi$ if

$$
\left\langle\left(\Delta Q_{N}(\varphi)\right)^{2}\right\rangle<\frac{1}{4}\left\langle F_{N}\right\rangle=\frac{1}{4}\left\langle\left[a^{N}, a^{+N}\right]\right\rangle
$$

where $\Delta Q_{N}(\varphi) \equiv Q_{N}(\varphi)-\left\langle Q_{N}(\varphi)\right\rangle$. It is easy to get [13]

$$
\left\langle\left(\Delta Q_{N}(\varphi)\right)^{2}\right\rangle=\frac{1}{4}\left\langle F_{N}\right\rangle+\left\langle:\left(\Delta Q_{N}(\varphi)\right)^{2}:\right\rangle
$$

with

$$
\left\langle:\left(\Delta Q_{N}(\varphi)\right)^{2}:\right\rangle=\frac{1}{2}\left\{\left\langle a^{+N} a^{N}\right\rangle+\Re\left[e^{-i 2 N \varphi}\left\langle a^{2 N}\right\rangle\right]-2\left(\Re\left[e^{-i N \varphi}\left\langle a^{N}\right\rangle\right]\right)^{2}\right\}
$$

and

$$
\left\langle F_{N}\right\rangle=\sum_{q=1}^{N} \frac{N ! N^{(q)}}{(N-q) ! q !}\left\langle a^{+(N-q)} a^{N-q}\right\rangle
$$

where :...: denotes a normal ordering of the operators and $N^{(q)}=N(N-1) \ldots(N-q+1)$.

For convenience, the squeezing degree is examined by a function $S$ defined as

$$
S=\frac{4\left\langle:\left(\Delta Q_{N}(\varphi)\right)^{2}:\right\rangle}{\left\langle F_{N}\right\rangle}
$$

in terms of which the state is said to be amplitude $N$ th power squeezed in the direction $\varphi$ if $-1 \leq S<0$. We choose the real axis along the direction of $\xi$ allowing to treat $\xi$ as a real number. In the fan-state, we have [15]

$$
\left\langle a^{+l} a^{m}\right\rangle_{k}=\frac{\xi^{(l-m)}}{D_{k}\left(\xi^{2}\right)} I\left(\frac{l-m}{2 k}\right) \sum_{n=0}^{\infty} \frac{\theta(2 k n-m) \xi^{4 k n} J_{k}\left(n+\frac{l-m}{2 k}\right) J_{k}(n)}{(2 k n-m) ! f(2 k n)(!)^{2 k} f(2 k n+l-m)(!)^{2 k}}
$$

where $\langle\ldots\rangle_{k} \equiv{ }_{F}\langle\xi ; 2 k, f|\ldots| \xi ; 2 k, f\rangle_{F}$. The function $I(x)$ equals unity if $x$ is an integer and zero otherwise. The step function $\theta(2 k n-m)$ can be removed and replaced in the 
summation $n=0$ by $n=n_{\min }$ with $n_{\min }$ equal to the integer part of $(m+2 k-1) / 2 k$. The properties of $J_{k}(n)$ can be given in the form

$$
J_{k}(n)= \begin{cases}2 k & \text { if } n \text { even integers } \\ 0 & \text { if } n \text { odd integers }\end{cases}
$$

and

$$
J_{k}(n) J_{k}\left(n+n^{\prime}\right)=\left\{\begin{array}{ll}
2 k^{2}\left(1+(-1)^{n}\right) & \text { if } n^{\prime} \text { even integers } \\
0 & \text { if } n^{\prime} \text { odd integers }
\end{array} .\right.
$$

The general expression of the squeezing degree is derived analytically for arbitrary $\xi, k, N$ and $f$ in the form

$$
S=\frac{2\left\{\left\langle a^{+N} a^{N}\right\rangle_{k}-\left(\left\langle a^{N}\right\rangle_{k}\right)^{2}+\cos (2 N \varphi)\left(\left\langle a^{2 N}\right\rangle_{k}-\left(\left\langle a^{N}\right\rangle_{k}\right)^{2}\right)\right\}}{\sum_{q=1}^{N} \frac{N ! N(q)}{(N-q) ! q !}\left\langle a^{+(N-q)} a^{N-q}\right\rangle_{k}}
$$

with

$$
\left\langle a^{N}\right\rangle_{k}=\frac{\xi^{-N}}{D_{k}\left(\xi^{2}\right)} I\left(-\frac{N}{2 k}\right) \sum_{n=0}^{\infty} \frac{\theta(2 k n-N) \xi^{4 k n} J_{k}\left(n-\frac{N}{2 k}\right) J_{k}(n)}{(2 k n-N) ! f(2 k n)(!)^{2 k} f(2 k n-N)(!)^{2 k}}
$$

and

$$
\left\langle a^{+N} a^{N}\right\rangle_{k}=\frac{2 k^{2}}{D_{k}\left(\xi^{2}\right)} \sum_{n=0}^{\infty} \frac{\theta(2 k n-N) \xi^{4 k n}\left(1+(-1)^{n}\right)}{(2 k n-N) !\left[f(2 k n)(!)^{2 k}\right]^{2}} .
$$

Since $\left\langle a^{+N} a^{N}\right\rangle_{k}$ is always positive and $\left\langle a^{2 N}\right\rangle_{k} \neq 0$ if $N$ is even and $\left\langle a^{2 N}\right\rangle_{k}=0$ if $N$ is odd, so that, the function $S$ in (14) may becomes negative only if $N$ is even, in which case squeezing is possible.

In what follows we consider separately the linear case which corresponds to the light field and the nonlinear case which may be associated with the vibrational motion of the trapped ion.

\section{LINEAR CASE}

For an arbitrary $N$, Eqs.(15)-(16) reduce to

$$
\left\langle a^{N}\right\rangle_{k}=\frac{\xi^{-N}}{D_{k}\left(\xi^{2}\right)} I\left(-\frac{N}{2 k}\right) \sum_{n=0}^{\infty} \frac{\theta(2 k n-N) \xi^{4 k n} J_{k}\left(n-\frac{N}{2 k}\right) J_{k}(n)}{(2 k n-N) !}
$$

and

$$
\left\langle a^{+N} a^{N}\right\rangle_{k}=\frac{2 k^{2}}{D_{k}\left(\xi^{2}\right)} \sum_{n=0}^{\infty} \frac{\theta(2 k n-N) \xi^{4 k n}\left(1+(-1)^{n}\right)}{(2 k n-N) !} .
$$

We note that in (17)-(18) for a given $k,\left\langle a^{N}\right\rangle_{k}=\xi^{4 k}$ if $N=4 k$ and $\left\langle a^{N}\right\rangle_{k}=0$ if $N \neq 4 k$. Hence, $\left\langle a^{+N} a^{N}\right\rangle_{k} \geq\left\langle a^{2 N}\right\rangle_{k} \geq\left(\left\langle a^{N}\right\rangle_{k}\right)^{2}$ if $N \neq 2 k$ and the function $S$ is positive resulting in no squeezing. For $N=2 k$, the squeezing is possible and may occur along $N$ directions, as will be shown explicitly below for $k=1$ and 2 . 
For $k=1(N=2)$ we have obtained

$$
S_{\varphi, N=2}^{(k=1)}=\frac{\xi^{4}\left[\cosh \left(\xi^{2}\right)-\cos \left(\xi^{2}\right)+D_{1} \cos (4 \varphi)\right]}{2 \xi^{2}\left(\sinh \left(\xi^{2}\right)-\sin \left(\xi^{2}\right)\right)+D_{1}}
$$

and squeezing occurs whenever

$$
\cos (4 \varphi)<h(|\xi|)=\frac{\cos \left(\xi^{2}\right)-\cosh \left(\xi^{2}\right)}{D_{1}} \leq 0
$$

with

$$
D_{1}=\cosh \left(\xi^{2}\right)+\cos \left(\xi^{2}\right) .
$$

The function $h(|\xi|)$ equals zero at $\xi=0$ and decreases when $|\xi|$ increases. There is no squeezing for $|\xi| \geq \xi_{c}=1.25331$ for which $h(|\xi|) \leq-1$ and no $\varphi$ can be found to make $S_{\varphi, N=2}^{(k=1)}$ negative. Fig. 1 is a $3 \mathrm{D}$ plot of $S_{\varphi, N=2}^{(k=1)}$ as a function of $|\xi|$ and $\varphi$. A maximal squeezing occurs simultaneously along the two directions $\varphi=\frac{(2 n+1) \pi}{4}$ with $n=0,1$. The two coexistent directions of squeezing are shown by a polar plot of $S_{\varphi, N=2}^{(k=1)}$ (Fig. 2) at $|\xi|=0.8$ which looks like a flower . The small wings correspond to squeezing, the big ones to stretching.

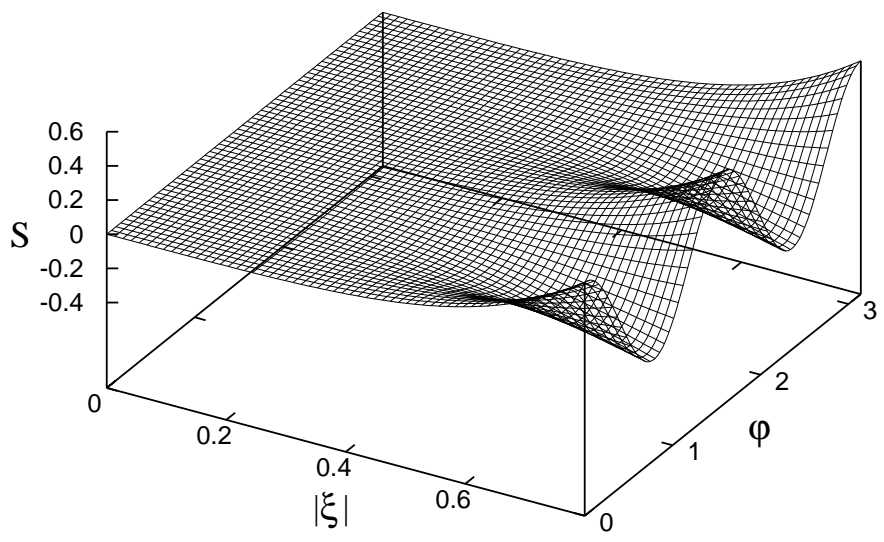

Fig. 1. The $S \equiv S_{\varphi, N=2}^{(k=1)}$ as a function of $|\xi|$ and $\varphi$ showing two directions of squeezing.

For $k=2(N=4)$ we have obtained

$$
S_{\varphi, N=4}^{(k=2)}=\frac{\xi^{8}}{4} \frac{\cosh \left(\xi^{2}\right)+\cos \left(\xi^{2}\right)-2 \cosh \left(\frac{\xi^{2}}{\sqrt{2}}\right) \cos \left(\frac{\xi^{2}}{\sqrt{2}}\right)+D_{2} \cos (8 \varphi)}{3 \xi^{4}\left[\cosh \left(\xi^{2}\right)-\cos \left(\xi^{2}\right)-2 \sinh \left(\frac{\xi^{2}}{\sqrt{2}}\right) \sin \left(\frac{\xi^{2}}{\sqrt{2}}\right)\right]+C+2 B-D_{2}},
$$

with

$$
D_{2}=\cosh \left(\xi^{2}\right)+\cos \left(\xi^{2}\right)+2 \cosh \left(\frac{\xi^{2}}{\sqrt{2}}\right) \cos \left(\frac{\xi^{2}}{\sqrt{2}}\right)
$$




$$
\begin{gathered}
A=\xi^{2}\left[\sinh \left(\xi^{2}\right)-\sin \left(\xi^{2}\right)+\sqrt{2}\left(\sinh \left(\frac{\xi^{2}}{\sqrt{2}}\right) \cos \left(\frac{\xi^{2}}{\sqrt{2}}\right)-\sin \left(\frac{\xi^{2}}{\sqrt{2}}\right) \cosh \left(\frac{\xi^{2}}{\sqrt{2}}\right)\right)\right], \\
B=3 \xi^{4}\left[\cosh \left(\xi^{2}\right)-\cos \left(\xi^{2}\right)-2 \sinh \left(\frac{\xi^{2}}{\sqrt{2}}\right) \sin \left(\frac{\xi^{2}}{\sqrt{2}}\right)\right]+6 A+2 D_{2}
\end{gathered}
$$

and

$C=2 \xi^{6}\left(\sinh \left(\xi^{2}\right)+\sin \left(\xi^{2}\right)-\sqrt{2}\left[\sinh \left(\frac{\xi^{2}}{\sqrt{2}}\right) \cos \left(\frac{\xi^{2}}{\sqrt{2}}\right)+\sin \left(\frac{\xi^{2}}{\sqrt{2}}\right) \cosh \left(\frac{\xi^{2}}{\sqrt{2}}\right)\right]\right)$.

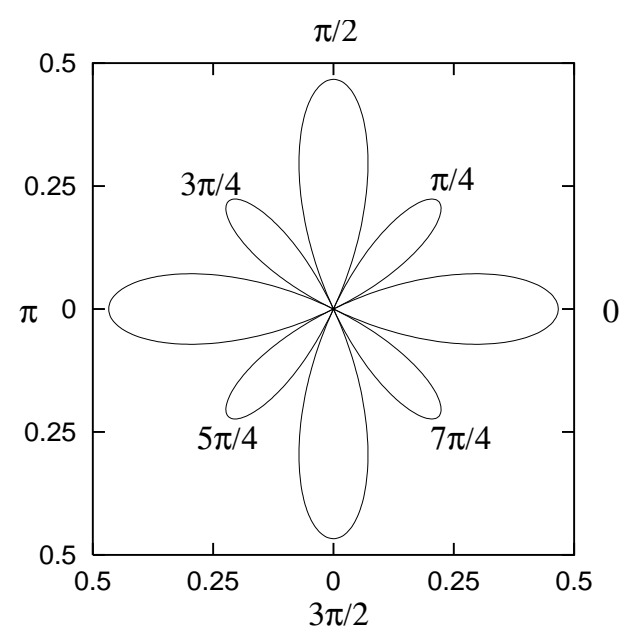

Fig. 2. The polar plots of the $S_{\varphi, N=2}^{(k=1)}$ for $|\xi|=0.8$.

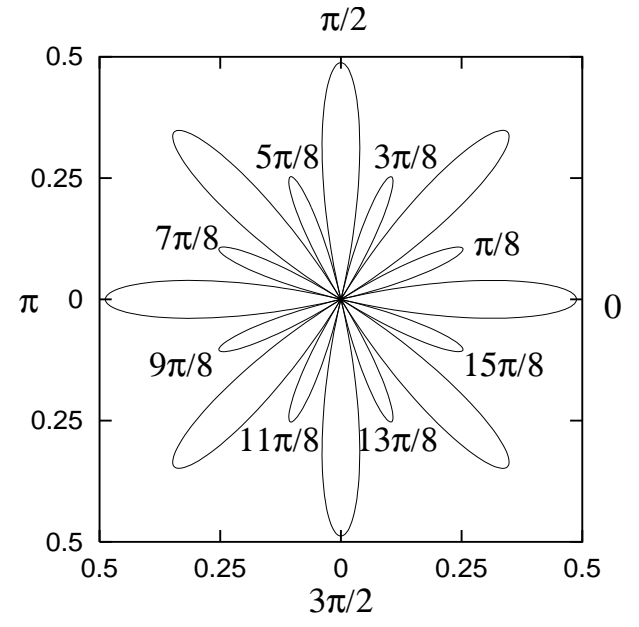

Fig. 3. The polar plots of the $S_{\varphi, N=4}^{(k=2)}$ for $|\xi|=1.25$.

The maximal squeezing occurs along the four directions $\varphi=\frac{(2 n+1) \pi}{8}$ with $n=$ $0,1,2,3$. Fig. 3 is a polar plot of $S_{\varphi, N=4}^{(k=2)}$ at $|\xi|=1.25$ as a function of $\varphi$. The four directions of squeezing are watched.

In general, for a given $k$, the Hillery-type N-powered amplitude squeezing depends on $\varphi$ only if $N=2 k$ in which case squeezing is possible. The number of squeezing directions scales precisely as $2 k$.

\section{NONLINEAR CASE}

In this section, we consider the Hillery-type N-powered amplitude squeezing of the vibrational motion of the trapped ion. In Refs.19-20, the specific function $f$ and the quantity $\xi$ are

$$
f(n+2 k)=\frac{n ! L_{n}^{2 k}\left(\eta^{2}\right)}{(n+2 k) ! L_{n}^{0}\left(\eta^{2}\right)}, \quad \quad \xi^{2 k}=-\frac{e^{i \phi} \Omega_{0}}{(i \eta)^{2 k} \Omega_{1}}
$$

where $L_{n}^{m}(x)$ is the $n^{\text {th }}$ generalized polynomial in $x$ for parameter $m, \eta$ is the Lamb-Dicke parameter, $\phi=\phi_{1}-\phi_{0}$ with $\phi_{0}\left(\phi_{1}\right)$ the phase of the driving laser which is resonant with (detuned to the $2 k^{\text {th }}$ lower sideband of) the electronic transition of the ion, and $\Omega_{0,1}$ the pure electronic transition Rabi frequencies. In the linear fan-states, there are two physical 
parameters $k$ and $\xi$. But in the case of the driven trapped ion, there are more physical parameters: The $\Omega_{0,1}$ which are controllable by the driving laser fields and $\eta$ which is controllable by trapping potential. Using (14-16) and (27), we can derive the Hillery-type N-powered amplitude squeezing for arbitrary $N$ and $k$. For the specific nonlinear function $f$ in (27), the simulation shows that for a given $k$ the Hillery-type is squeezed only for $N=2 k$ in some range of the values of $\xi^{2}$ and $\eta^{2}$. In the trapped ion, by controlling the Lamb-Dicke parameter and the pure transition Rabi frequencies, the higher order squeezing in nonlinear fan-states may occur along $N$ directions. Fig. 4 plots $S_{\varphi, N=2}^{(k=1)}$ for $\varphi=\frac{\pi}{4}$ and $\eta^{2}=0.05$ as a function of $\xi^{2}$. In this case, the squeezing exists for $\xi$ such that $0<\xi^{2}<1.01$ and maximal squeezing at $\xi^{2}=0.67$. Fig. 5 plots $S_{\varphi, N=4}^{(k=2)}$ for $\varphi=\frac{\pi}{8}$ as a function of $\xi^{2}$. We plot for $\eta^{2}=0.1716$ and $\eta^{2}=0.1722$ in order to show that the squeezing exists in some range of the values of $\xi^{2}$ and degrees of the squeezing depend on the changing of $\eta^{2}$. In general, for a given $k$ and arbitrary values of even orders $N=2 k$, we could choose $\eta$ and $\xi$ such that $\left\langle a^{2 N}\right\rangle_{k}>\left\langle a^{+N} a^{N}\right\rangle_{k}$ and squeezing appears equally maximal at

$$
\varphi_{\max }=\frac{\pi}{2 N}(1+2 n) \quad \text { with } n=0,1, \ldots, N-1 .
$$

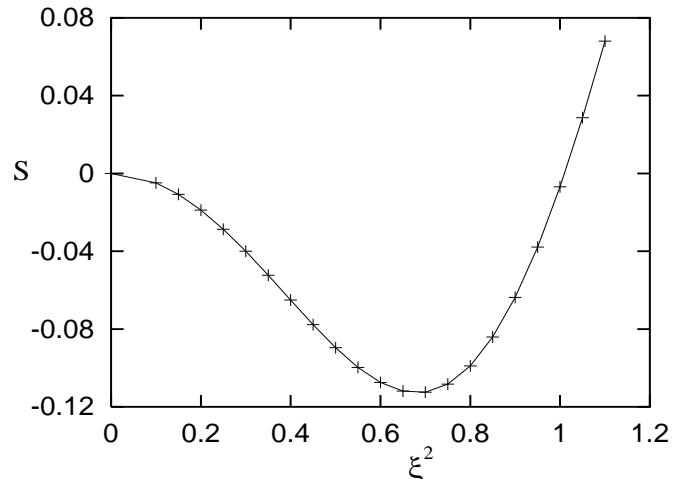

Fig. 4. The $S \equiv S_{\varphi, N=2}^{(k=1)}$ as a function of $\xi^{2}$ for $\varphi=\frac{\pi}{4}$ and $\eta^{2}=0.05$.

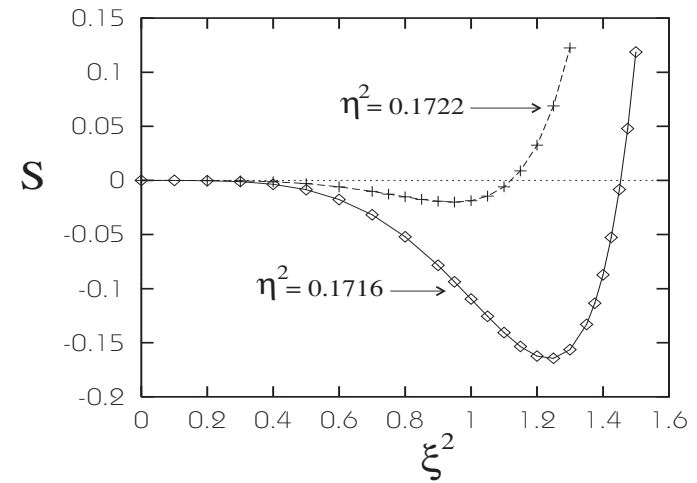

Fig. 5. The $S \equiv S_{\varphi, N=4}^{(k=2)}$ as a function of $\xi^{2}$ for $\varphi=\frac{\pi}{8}, \eta^{2}=0.1716$ and $\eta^{2}=0.1722$.

\section{CONCLUSION}

We have investigated the Hillery-type N-powered amplitude squeezing for the linear ( the light field) and nonlinear fan-states (the trapped ion). The formulas derived above are applicable to arbitrary $k, N$ and $f$ and the number of squeezing directions does not depend on whether the state is linear or nonlinear. Given $k$, the squeezing is possible for $N=2 k$ in both cases and appears simultaneously along $N$ different directions. The squeezing directions in Hillery-type of higher order for the linear fan-states case occur along $N$ directions (see (28)). For the nonlinear fan-states as the trapped ion, the number of squeezing directions is the same as for the linear fan-states but the degree of the squeezing themselves are adjustable by controlling the parameters of the laser-driven trapped-ion system (see Fig. 5 for example). 


\section{ACKNOWLEDGMENTS}

The author is grateful to Professor Nguyen Ba An for his kind guidance and useful discussions .

\section{REFERENCES}

1. V. V. Dodonov, J. Opt. B: Quantum Semiclass. Opt., 4 (2002) R1

2. D. F. Wall, Nature, 306 (1983) 141.

3. J. Janszky, et al., Phys. Rev. A, 53 (1996) 502.

4. J. Lee, et al., Phys. Rev. A, 62 (2000) 32305.

5. W. Vogel, Phys. Rev. Lett. 84 (2000) 1849.

6. V. N. Gorbachev, A. I. Zhiliba and A. I. Trubilko, J. Opt. B: Quantum Semiclass Opt. 3 (2001) S25.

7. A. L. de Souza Silva, S. S. Mizrahi and V. V. Dodonov, J. Russ. Laser Res., 6 (2001) 534.

8. D. Stoler, Phys. Rev. D, 1 (1990) 3217; 4 (1971) 1935.

9. C. K. Hong and L. Mandel, Phys. Rev. Lett., 54 (1985) 323.

10. M. Hillery, Opt. Commun., 62 (1987) 135.

11. You - bang Zhan, Phys. Lett. A, 160 (1991) 498.

12. Si - De Du and Chang - De Gong, Phys. Lett. A, 168 (1992) 296.

13. Nguyen Ba An, Phys. Lett. A, 234 (1997) 45.

14. Nguyen Ba An, Phys. Lett. A, 72 (2001) 284.

15. Nguyen Ba An and Truong Minh Duc, Int. J. Mod. Phys., B16 (2002) 519.

16. Xiao - Ming Liu, J. Phys. A: Math. Gen., 32 (1999) 8685.

17. Nguyen Ba An and Truong Minh Duc, J. Phys. A: Math. Gen., 35 (2002) 4749.

18. V. I. Manko, G. Marmo, A. Porzio, S. Somimeno, F. Zaccaria, Phys. Rev. A 62 (2002) 053407; E. C. G. Sudarshan Int. J. Theor. Phys., 32 (1993) 1069.

19. Nguyen Ba An, Chinese J. Phys. 39 (2001) 594.

20. V. I. Manko, G. Marmo, A. Porzio, S. Solimeno and F. Zaccaria, Phys. Rev. A, 62 (2000) 053407 . 